\title{
Gut Microbiota-Dependent Modulation of Energy Metabolism
}

\author{
Christina N. Heiss Louise E. Olofsson \\ Wallenberg Laboratory, Department of Molecular and Clinical Medicine, Institute of Medicine, University of \\ Gothenburg, Gothenburg, Sweden
}

\section{Keywords}

Microbiota - Energy balance - Obesity - Leptin .

Hypothalamus - Metabolites - Microbial products .

Short-chain fatty acids $\cdot$ Secondary bile acids

\begin{abstract}
The gut microbiota has emerged as an environmental factor that modulates the host's energy balance. It increases the host's ability to harvest energy from the digested food, and produces metabolites and microbial products such as shortchain fatty acids, secondary bile acids, and lipopolysaccharides. These metabolites and microbial products act as signaling molecules that modulate appetite, gut motility, energy uptake and storage, and energy expenditure. Several findings suggest that the gut microbiota can affect the development of obesity. Germ-free mice are leaner than conventionally raised mice and they are protected against dietinduced obesity. Furthermore, obese humans and rodents have an altered gut microbiota composition with less phylogeneic diversity compared to lean controls, and transplantation of the gut microbiota from obese subjects to germ-free mice can transfer the obese phenotype. Taken together, these findings indicate a role for the gut microbiota in obesity and suggest that the gut microbiota could be targeted to improve metabolic diseases like obesity. This review focuses on the role of the gut microbiota in energy balance regulation and its potential role in obesity.
\end{abstract}

(c) 2017 S. Karger AG, Basel

\section{KARGER}

๑ 2017 S. Karger AG, Basel

E-Mail karger@karger.com

www.karger.com/jin

\section{The Host-Microbiota Symbiosis}

At birth, the human body becomes colonized by microbes, leading to colonization of practically all parts of the human body that are in direct contact with the external environment. Recently, it was estimated that $3.8 \times$ $10^{13}$ microbes are present in an average $70-\mathrm{kg}$ man [1]. The intestine, particularly the colon, has the highest abundance of microbes [2]. In humans, the gut microbiota composition changes during the first year of life, and a more mature microbiota composition has been acquired by 1 year of age [3]. This maturation is food-dependent and requires cessation of breast-feeding rather than introduction of solid food. Several factors influence the adult microbiota composition, including diet, use of antibiotics, hygiene, and host's genetics [4,5]. Dietary interventions with plant- and animal-based diets have shown that the diet can rapidly and reproducibly change the microbiota composition [4]. The importance of the host's genetics on the microbiota composition has been highlighted in a study on the TwinsUK population [5].

Most of the microbes colonizing the human intestine belong to the phyla Firmicutes and Bacteroidetes and, to a lesser extent, Actinobacteria, Proteobacteria, Verrucomicrobia, Fusobacteria, and Euryarchaeota [3]. The environment varies along the intestine; 2 examples being the extreme differences in $\mathrm{pH}$ and oxygen concentration between the stomach and the colon. Such environmental

Dr. Louise E. Olofsson

Wallenberg Laboratory, Sahlgrenska University Hospital Bruna Stråket 16

SE-413 45 Gothenburg (Sweden)

E-Mail Louise.Olofsson@wlab.gu.se 
factors affect the ability of different bacteria to colonize the gut, leading to regional variations in the microbiota composition [6]. For example, due to the higher oxygen concentration in the small intestine than in the colon, the upper small intestine is dominated by facultative anaerobic and aerotolerant microbes, while the colon is dominated by strictly anaerobic microbes [6].

Due to the close interaction between the host and the gut microbiota, the microbiota can influence the host's physiology and metabolism. The combined genome of the colonizing microbes has been estimated to contain at least 100 times as many genes as the human genome, and microbial products and metabolites can directly affect the host's physiology [2, 7]. The microbiota has many important roles including maturation of the host's immune system, improvement of intestinal barrier function, and preventing colonization of pathogenic microbes. Thus, the commensal microbes live in symbiosis with their host. In addition to protecting the host against pathogens, the commensal microbes can also affect the host's energy homeostasis and play a role in obesity.

\section{Changes in Microbiota Composition in Obesity}

Metabolic diseases such as obesity are associated with alterations in the gut microbiota composition in both humans and rodents. Some studies have found an increased Firmicutes-to-Bacteroides ratio in obese humans and rodents compared to lean controls [8-10], but other studies have failed to observe such a difference [11]. However, more consistently, most studies report a decreased phylogeneic diversity and a reduced number of bacterial genes present in obese versus lean subjects $[10,12]$. Furthermore, low microbial richness also correlates with other metabolic parameters such as serum insulin, HOMA insulin resistance, and free fatty acid and triglyceride levels in the plasma [12].

The potential causative role of the gut microbiota in obesity is supported by studies showing that the obese phenotype can be transferred by gut microbiota transplantation [9, 13]. Turnbaugh et al. [9] showed that the colonization of germ-free (GF) mice with cecal content from an obese donor mouse led to an increased weight gain in the recipient mice compared to colonization with cecal content from a lean donor mouse. Furthermore, they showed that colonization with a microbiota from an obese donor mouse resulted in decreased fecal energy excretion, indicating that the gut microbiota from obese donors has an increased capacity to harvest energy from the diet. The transplantation of human fecal microbiota from lean and obese donors to recipient mice also transferred the obese phenotype [13]. While the fat mass of mice transplanted with gut microbiota from obese donors had increased 2 weeks after colonization, that of mice transplanted with gut microbiota from lean donors did not increase. Furthermore, cohousing mice that received a gut microbiota from obese donors with mice that received a gut microbiota from lean donors led to a reduced increase in adiposity in the mice colonized with the gut microbiota from the obese donors [13]. The cecal microbiota in the mice colonized with gut microbiota from obese donors changed after cohousing, becoming more similar to that in mice colonized with gut microbiota from lean donors. In particular, the invasion by specific members of Bacteroidetes from the "lean microbiota" to the mice colonized with the "obese microbiota" was associated with the rescue of the obese phenotype. In contrast, the cecal microbiota in mice colonized with a gut microbiota from lean donors remained stable after cohousing. Collectively, these findings suggest that the gut microbiota plays a role in the regulation of energy balance and the development of obesity.

\section{The Gut Microbiota and the Host's Energy Balance}

GF mice are lean compared to conventionally raised (CONV-R) mice, and colonization of GF mice leads to rapid weight gain and an increase in adiposity [14]. Part of this increase in body weight and adiposity can be explained by a microbe-mediated increase in energy uptake. Microbes residing in the gut metabolize otherwise-indigestible complex carbohydrates by fermentation, leading to the production of short-chain fatty acids (SCFAs). SCFAs can be taken up in the gut, and thereby act as an energy source, causing an increased energy harvest from the ingested food. This increased energy harvest has been estimated to account for $10 \%$ of the energy intake in people living in the Western world [15]. Colonization experiments further highlight the importance of the increased energy harvest; it has been shown that colonization of GF mice leads to increased body weight and adiposity, even though the colonized mice have a decreased food intake and an increased energy expenditure compared to GF mice [14]. Taken together, these results suggest that the SCFAs produced by fermentation in humans and mice account for a substantial part of the energy uptake. Nevertheless, in addition to the role of the gut microbiota in energy uptake, there is evidence that the gut microbiota 
directly modulates the host's regulation of the energy balance. GF mice are protected against diet-induced obesity (DIO) when given a Western diet containing a low amount of complex carbohydrates [16]. Thus, even when the fermentation rate is low, the presence of a gut microbiota leads to a positive energy balance. However, the microbiota-diet interaction is complex, and the resistance to DIO in mice lacking a microbiota depends on the composition and fat source of the high-fat diet (HFD) [17]. Increased energy expenditure, as well as increased fecal fat content and energy excretion, contribute to the DIO resistance in GF mice when fed a lard-based HFD. In line with these results showing an increased energy expenditure in GF mice, it was recently shown that mice lacking a microbiota i.e., GF or antibiotic-treated mice, have increased browning in both the inguinal subcutaneous and the perigonadal visceral adipose tissue [18]. Both GF and antibiotic-treated mice had increased expression of browning markers including uncoupled protein 1 (UCP1 ) as well as increased oxygen consumption in isolated adipocytes [18]. Collectively, these results suggest that increased energy expenditure explains at least parts of the resistance to DIO in GF mice.

\section{The Gut-Brain Axis}

The brain receives information from peripheral organs such as the intestine and uses this information to regulate the energy balance. There is a bidirectional communication between the gut and the brain, known as the gut-brain axis. The brain can signal to the gut via efferent vagal signaling as well as via neuroendocrine pathways. The brain's communication with the gut microbes can be direct, i.e., when neurotransmitters, including catecholamines, 5 -hydroxytryptamine (5-HT), and $\gamma$-aminobutyric acid (GABA), are sensed by the microbes, or indirect, through the influence on the intestinal milieu. The intestinal milieu can be affected by vagal efferent nerves that regulate intestinal functions such as gut motility, acid and mucus secretion, intestinal barrier function as well as mucosa immune response, which thereby influence the gut microbiota composition and function [19].

The gut communicates with the brain via blood-borne substances or afferent spinal and vagal nerves, allowing the gut and the gut microbiota to signal directly to the brain. Microbial products and microbially produced metabolites can act as signaling molecules and regulate the secretion of hormones from intestinal enteroendocrine cells. These hormones include peptide YY (PYY) [20] and glucagon-like peptide-1 (GLP-1) [21, 22], both of which receptors are expressed in regions of the brains which regulate energy balance, including the hypothalamus $[20,23]$.

\section{Leptin, Microbiota, and Hypothalamic Energy Balance Regulation}

As mentioned above, the hypothalamus is a part of the brain that is important in the regulation of energy balance. Leptin, a hormone produced in the adipose tissue, acts on the long form of the leptin receptor which is highly expressed in hypothalamic neurons. Leptin is secreted from the adipose tissue in proportion to the amount of fat in the body, and can thus communicate the energy stores to the brain [24]. The neurons expressing proopiomelanocortin (POMC) and the neurons coexpressing agoutirelated protein (AgRP) and neuropeptide Y (NPY) are among the most studied leptin-targeted neurons. Both of these types of neurons are located in the hypothalamic arcuate nucleus (ARC). Leptin inhibits the orexigenic AgRP neurons and activates the anorexigenic POMC neurons resulting in decreased food intake and increased energy expenditure. Both AgRP and POMC neurons act on melanocortin 4 receptor (MC4R)-expressing neurons in the paraventricular nucleus [24].

Most obese subjects and DIO mice have high levels of circulating leptin, but are leptin-resistant and have an impaired response to leptin. Thus, the leptin-mediated decrease in food intake and increase in energy expenditure is diminished. Hypothalamic leptin resistance is observed within a few days after switching to an HFD in rodents, before any significant weight difference is observed. Therefore, leptin resistance is believed to play a central role in the pathogenesis of obesity. Interestingly, GF mice have been shown to have improved leptin sensitivity when compared to CONV-R mice [21]. Intraperitoneal injection of leptin results in a greater weight loss in chowfed GF mice when compared to CONV-R mice. Furthermore, suppressor of cytokine signaling 3 (SOCS3), an inhibitor of intracellular leptin signaling, is increased in CONV-R mice compared to in GF mice, potentially contributing to the difference in leptin sensitivity [21].

Leptin resistance can be caused by different mechanisms including diminished intracellular signaling, impaired transport over the blood-brain barrier (BBB) as well as hypothalamic inflammation and neuronal injury. Switching to an HFD leads to hypothalamic inflammation before any weight gain is observed and results sug- 
gest that such inflammation can cause leptin resistance $[25,26]$. Hypothalamic inflammation is characterized by increased number and reactivity of microglia and astrocytes in the mediobasal hypothalamus, increased expression of inflammatory mediators, and neuronal injury. The role of the gut microbiota in diet-induced hypothalamic inflammation is not known. However, findings suggest that the properties of the microglia differ in GF and CONV-R mice. Erny et al. [27] showed that GF mice have immature microglia cells which are unable to respond normally to lipopolysaccharide (LPS) stimuli. Furthermore, they showed that microglia need constant stimulation from the gut microbiota to remain mature; a 4-week antibiotic treatment led to immature microglia cells with an impaired response to LPS. Administration of SCFAs to the GF mice restored the immature microglia phenotype, and mice lacking the SCFA receptor GPR43 had severely malformed microglia which mimicked the microglia in GF mice.

The mediobasal hypothalamus, including the ARC, is uniquely located close to the median eminence, in a region with an incomplete BBB. Due to this location, neurons in the mediobasal hypothalamus can more readily sense substances in the blood such as microbial products and metabolites as well as microbially induced hormonal signals. The gut microbiota may also regulate the brain's access to circulating factors by affecting the BBB permeability. Braniste et al. [28] showed that GF mice had increased $\mathrm{BBB}$ permeability when compared to CONV-R mice. This difference was already observed in the embryonic state, suggesting that the maternal gut microbiota can influence the development of the BBB in the embryo. GF mice had disorganized tight junctions and reduced expression of the tight-junction proteins occludin and claudin-5. Furthermore, colonization of GF mice led to decreased BBB permeability, which was associated with an increased expression of occludin [28]. Taken together, the microbiota may modulate the hypothalamic regulation of energy balance for example by affecting leptin sensitivity, microglia function, and BBB permeability.

\section{Signaling via Microbial Metabolites}

The gut microbiota affects the host's energy metabolism via its microbial products and metabolites (Fig. 1). There is a strong link between the diet, the gut microbiota, and the effects on the host's metabolism [29, 30]. As mentioned above, certain dietary components will favor some microbes but not others, and the diet will therefore strongly influence the gut microbiota composition. Additionally, the diet composition will also determine which metabolites are produced by the gut microbiota.

\section{SCFA Signaling}

The SCFAs, butyrate, propionate, and acetate, produced by fermentation, are among the most studied bacterial metabolites. Besides being an energy source, these SCFAs can also act as signaling molecules, binding to their G protein-coupled receptors GPR43 and GPR41 [31]. GPR43 is mainly expressed in immune cells and adipocytes, and has been suggested to play a major role in energy homeostasis. GPR43-deficient mice are obese when fed a chow diet, and mice overexpressing GPR43 specifically in the adipose tissue are resistant to DIO when fed an HFD [32]. SCFA-mediated activation of GPR43 in the adipose tissue suppresses insulin signaling and decreases lipid storage in the adipocytes, as well as increases the oxidation of lipids in other tissues, resulting in increased energy expenditure [32]. The body weight phenotypes in mice overexpressing GPR43 in adipose tissue and mice lacking GPR43 require the presence of microbes [32].

In addition to GPR43, SCFAs also act on GPR41, which is highly expressed in the adipose tissue as well as in a subset of enteroendocrine cells in the gut epithelium $[31,33]$. While CONV-R mice lacking GPR41 are leaner than wild-type mice, such a weight difference is not observed when the mice are kept under GF conditions [33]. Activation of GPR41 in adipose tissue by SCFAs has been shown to stimulate leptin expression in vitro, and oral administration of propionate increases the circulating levels of leptin [34]. In line with these results, GPR41deficient mice had diminished circulating leptin levels in proportion to their fat mass, an effect that is abolished when the mice lack a microbiota [33]. In addition to these effects on leptin levels, the CONV-R GPR41-deficient mice had reduced expression of the Pyy gene, encoding for the enteroendocrine hormone PYY. PYY inhibits gut motility, and GPR41-deficient mice had a faster gut transit time, extracted fewer calories from a polysacchariderich chow diet, and excreted more SCFAs, resulting in the lean phenotype. Taken together, these findings suggest that microbially produced SCFAs act on both GPR 43 and GPR41, and thereby play major roles in the regulation of energy homeostasis.

The incretin hormone GLP-1 is encoded by the $G c g$ gene, and is mainly produced in the brainstem and in the L-cells in the intestine [21, 22]. SCFAs, including propionate, have been shown to stimulate GLP-1 secretion in 


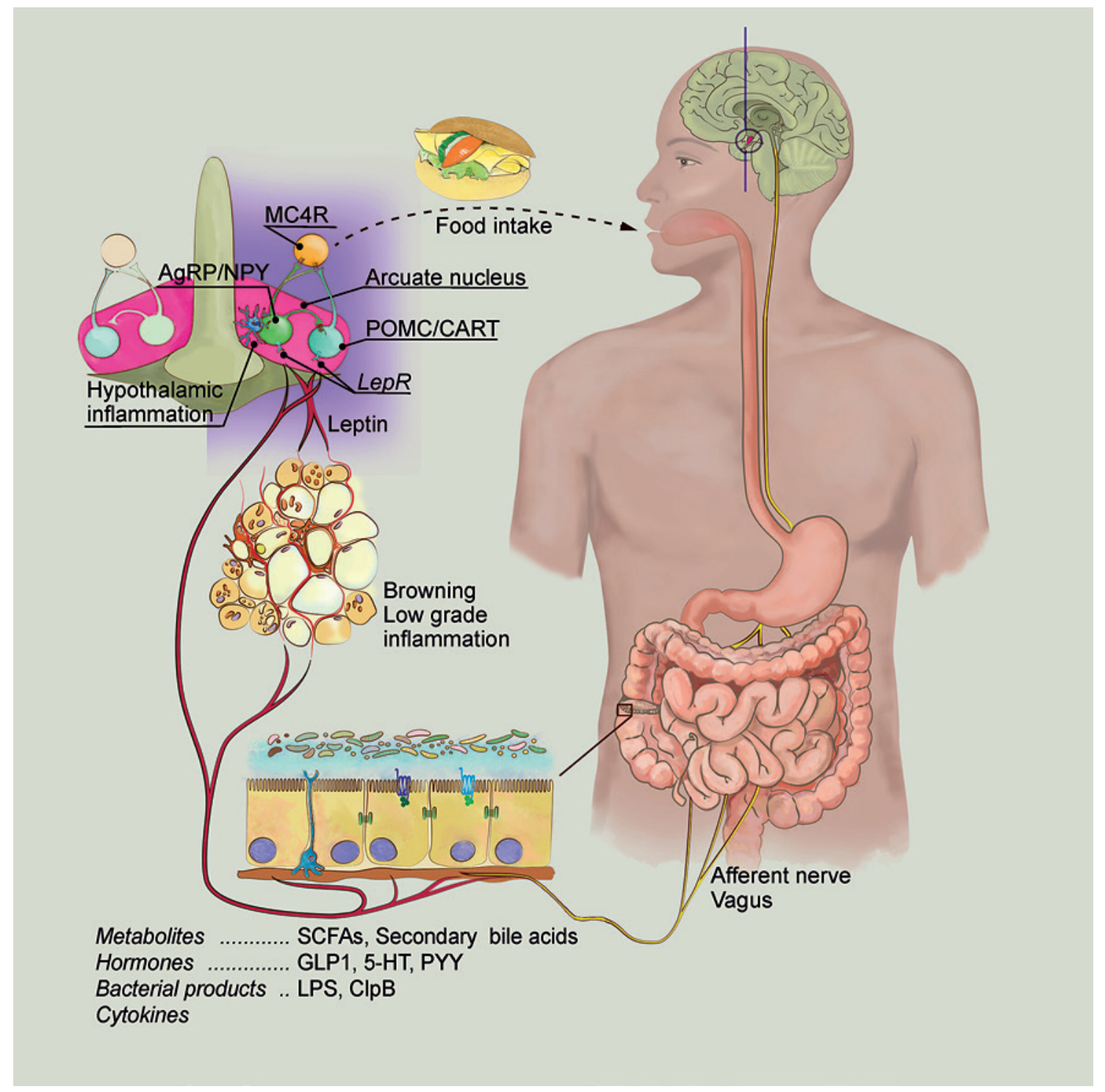

Fig. 1. Gut microbiota-dependent modulation of energy metabolism. Obese humans as well as obese mice have an altered gut microbiota when compared to lean controls, and this gut microbiota composition can transfer the obese phenotype when transplanted into a recipient mouse. Studies using GF, antibiotic-treated, and CONV-R wild-type and mutant mice have shown that the gut microbiota can modulate important processes in the regulation of energy balance. The diet strongly affects the gut microbiota composition and determines which metabolites are produced by the gut microbiota. These metabolites include SCFA and secondary bile acids, which, in turn, can bind to their receptors and thereby activate specific signaling pathways in the host. They can also regulate the secretion of hormones such as GLP-1, PYY, and leptin, which exert their effects in the brain via the circulation or by bind- ing to the vagal afferent nerves. The gut microbiota can also modulate the host's metabolism by microbial products including LPS, which causes low-grade inflammation, and ClpB, which can directly affect the POMC neuronal activity in the hypothalamus. The gut microbiota also has profound effects on intestinal barrier function, the immune system, and the immune response. Together, these signals affect food intake, gut motility, nutrient absorption, and energy utilization and expenditure. SCFAs, short-chain fatty acids; GLP-1, glucagon-like peptide-1; 5-HT, 5-hydroxytryptamine; PYY, peptide YY; LPS, lipopolysaccharide; LPR, leptin receptor; POMC, proopiomelanocortin; CART, cocaine- and amphetamine-regulated transcript; AgRP, agouti-related protein; NPY, neuropeptide Y; MC4R, melanocortin 4 receptor. 
colonic crypt cultures (murine L-cells), as well as to increase GLP-1 plasma levels in rodents via a GPR43-dependent mechanism [35]. However, the effects of SCFAs on GLP-1 secretion are complex and dependent on energy availability. Recent findings suggest that $G c g$ expression in colonic L-cells is upregulated when the colonocytes are energy-deprived. Since colonocytes use SCFAs as their primary energy source, a decrease in SCFAs leads to energy-deprived colonocytes [36] and higher Gcg expression. Thus, even though GF and antibiotic-treated mice have a low concentration of SCFAs in the cecal content due to reduced or absent fermentation, these mice have a high colonic $G c g$ expression and high plasma levels of GLP-1 [22]. Furthermore, administration of SCFAs decreases colonic $G c g$ expression in GF mice ex vivo, and feeding an HFD normalizes this expression in vivo [22], supporting the idea that the energy availability regulates the $G c g$ expression. While the increased GLP-1 in GF mice does not improve the incretin response, it leads to a slower gut transit time which allows a more efficient uptake of nutrients.

In addition to the effects of GLP-1 on gut transit, it also has anorexic effects [37]. Furthermore, studies in rodents have shown that intravenous administration of GLP-1 leads to increased whole-body oxygen consumption as well as increased body temperature [37]. GLP-1 has a half-life of approximately 1-2 min in the circulation due to rapid degradation by the enzyme dipeptidyl peptidase IV [22]. It is therefore believed that GLP-1 exerts much of its effects by binding receptors on vagal afferent nerves. Gastric vagal afferents nerves respond rapidly to intravenous GLP-1 administration, and this response is diminished by pretreatment with the GLP-1 receptor antagonist Exendin 9-39, indicating that the effect of GLP-1 is mediated by the GLP-1 receptor located on the vagal afferent nerves [23]. However, the GLP-1 receptor is also expressed in the hypothalamus and the brainstem, regions of the brain involved in the regulation of energy homeostasis, and GLP-1 could thus also directly target the GLP-1 receptor-expressing neurons $[21,38]$. Taken together, the gut microbiota produces SCFAs that act as signaling molecules, and thereby modulate the secretion of GLP-1, PYY, and leptin, and affect the gut motility and the gut transit time, as well as the fat storage in the adipose tissue.

\section{Bile Acid Signaling}

In addition to producing SCFAs, the gut microbiota metabolizes primary bile acids to secondary bile acids. Primary bile acids are produced from cholesterol in the liver, and are secreted into the duodenum after a meal. The primary bile acids produced in humans and rodents differ; while chenodeoxycholic acid and cholic acid are produced in humans, cholic acid and muricholic acids are produced in rodents [39]. In the intestine, bile acids act as surfactants, facilitating the action of lipases on digested fat and enhancing fat absorption. However, in addition to these effects, bile acids can also act as signaling molecules by binding to their receptors farnesoid X receptor (FXR) and G-protein-coupled membrane receptor 5 (TGR5) [39]. When the primary bile acids are secreted into the intestinal lumen, the gut microbes get access to them and can metabolize them through deconjugation, dehydrogenation, dihydroxylation, and epimerization. Depending on the modification, the properties of bile acids change, which, in turn, modulates their signaling capacity. For example, while FXR's main ligands are the primary bile acids, chenodeoxycholic acid and cholic acid, TGR5 primarily binds to the secondary bile acids, lithocholic acid and deoxycholic acid [39]. Bile acids can signal via these receptors to regulate, for example, the synthesis of the bile acids themselves and the secretion of enteroendocrine hormones. As an example, activation of TGR5 in the enteroendocrine L-cells has been shown to induce GLP-1 release [40].

Bile acid synthesis is regulated by negative feedback through FXR activation. In rodents, cholic acid activates FXR signaling in the intestine and exerts a negative feedback on the bile acids synthesis. In contrast, tauro-conjugated beta- and alpha-muricholic acids act as an FXR antagonist and thereby diminish this negative feedback. Thus, CONV-R mice, which have reduced levels of tauroconjugated beta- and alpha-muricholic acids, have increased fibroblast growth factor 15 (Fgf15) expression in the intestine, reduced expression of cholesterol 7a-hydroxylase (Cyp7a1) in the liver, and a reduced enterohepatic bile acid pool compared to GF mice [41]. Furthermore, the microbiota affects the whole body metabolism via FXR signaling; while CONV-R wild-type mice develop DIO when fed an HFD, CONV-R Fxr ${ }^{-/-}$mice and GF mice are resistant to DIO [42]. CONV-R wild-type mice fed an HFD also develop adipose tissue inflammation and hepatic steatosis, but these phenotypes are not present in $\mathrm{Fxr}^{-/-}$mice or in GF mice fed an HFD. Moreover, FXR signaling changes the gut microbiota composition, and colonization of GF mice with cecal content from an HFDfed wild-type donor mouse leads to a greater weight gain than colonization of GF mice with cecal content from an HFD-fed $\mathrm{Frr}^{-/-}$donor mouse. Taken together, the microbiota metabolizes primary bile acids to secondary bile ac- 
ids, and these can signal via FXR and TGR5 to regulate the host's metabolism including the bile acid synthesis and GLP-1 secretion.

\section{5-Hydroxytryptamine Signaling}

Another important signaling molecule is 5-HT (also known as serotonin), which is produced by microbes and enteroendocrine cells in the intestine. Approximately $90 \%$ of the body's 5 -HT is present in the enterochromaffin cells in the intestine, where it regulates intestinal movement. 5-HT is released from the enterochromaffin cells upon intraluminal pressure, leading to activation of the receptors and peristaltic movement [43]. Gut-derived 5-HT has also been shown to be important for fastinginduced adaptation by promoting lipolysis in adipose tissue and gluconeogenesis in liver, thereby increasing the availability of energy for other organs in the body [44]. The gut microbiota is associated with a higher intestinal expression of tryptophan hydroxylase 1 (Tph1), the ratelimiting enzyme for the synthesis of 5-HT, and stimulation of BON cells (the human enterochromaffin cell model) with acetate and butyrate leads to increased expression of Tph1 in vitro [45]. Receptors for 5-HT are expressed in many types of cells in the intestine including the enterochromaffin cells, goblet cells, enterocytes, vagal and spinal afferent nerves, and enteric nerves [43]. Collectively, these results suggest that the gut microbiota affects 5-HT levels, which, in turn, regulate important functions such as gut motility and fasting-induced lipolysis and gluconeogenesis.

\section{Signaling via Microbial Products}

In addition to metabolites, the microbes can also affect the host by producing microbial products such as LPS and caseinolytic protease $\mathrm{B}(\mathrm{ClpB})$.

\section{LPS Signaling}

Metabolic diseases such as obesity and type 2 diabetes are characterized by a low-grade inflammation with increased circulating levels of LPS, i.e., endotoxemia [46]. Elevated LPS levels, similar to those observed after feeding an HFD for 4 weeks, could cause weight gain and insulin resistance by itself via a mechanism that is dependent on the LPS receptor, CD14 [46]. While both high-fat feeding and LPS administration caused increased expression of inflammatory mediators, macrophage infiltration in the white adipose tissue, and weight gain in wild-type mice, these effects were blunted in mice lacking CD14.
Antibiotic treatment abolished the HFD-induced endotoxemia, adipocyte hypertrophy, inflammation, and microphage infiltration in the white adipose tissue [47].

The microbiota-induced white adipose tissue inflammation depends on the dietary composition of the HFD. Feeding a lard-based HFD leads to a change in the gut microbiota composition, which, in turn, causes increased LPS plasma levels and inflammation in the adipose tissue (via Toll-like receptor signaling) [48]. In contrast, feeding an HFD based on fish oils leads to a different gut microbiota composition and does not cause adipose tissue inflammation to the same extent as when the HFD is lardbased. Interestingly, when recipient mice were transplanted with gut microbiota from lard-fed or fish oil-fed mice and then subsequently fed a lard-based HFD, the mice receiving gut microbiota from fish oil-fed mice had reduced weight gain and diminished adipose tissue inflammation compared to those receiving gut microbiota from lard-fed mice.

Evidence suggests that the increased circulating LPS levels after high-fat feeding could be the result of a more permeable intestinal barrier. Feeding an HFD increased the gut permeability by decreasing the expression of tightjunction proteins including zonula occludens 1 and occludin [47]. However, antibiotic treatment restored the expression of these tight-junction proteins, and the gut permeability in antibiotic-treated mice fed an HFD remained similar to that in chow-fed mice. There is also evidence that chylomicrons, formed in the intestine, facilitate the absorption of LPS in the intestine [49]. Intestinal epithelial cells internalize LPS, and transport it to the Golgi apparatus where it associates with newly formed chylomicrons and is released into the lacteals. Feeding an HFD increases the chylomicron formation, which, in turn, could increase the LPS absorption.

Taken together, these results suggest that LPS from the gut microbes could contribute to the development of metabolic diseases. Feeding an HFD alters the gut microbiota composition and increases LPS absorption, leading to increased circulating LPS levels.

\section{ClpB Signaling}

The gut microbiota has also been implicated in the appetite control by modulating hypothalamic POMC neuronal function via the bacterial product $\mathrm{ClpB}$ [50]. Colonic infusion of nutrients induces an initial exponential bacterial growth, which shifts after around $20 \mathrm{~min}$ to a stationary growth phase. The abundance of different $E$. coli proteins varies in the different growth phases. For example, ClpB, a bacterial protein mimetic of $\alpha$-melanocyte- 
stimulating hormone, increases in the stationary growth phase. Breton et al. [50] showed that $\mathrm{ClpB}$ can act directly on the hypothalamic POMC neurons and increase the firing of these neurons, and may thereby induce satiety. Thus, commensal microbes such as E. coli can regulate the host's satiety via its bacterial proteins, the abundance of which is associated with nutrient-induced bacterial growth.

\section{Summary}

In conclusion, the commensal gut microbes live in symbiosis with their host, affecting many important functions including the regulation of energy balance. The gut microbiota increases energy uptake and produces metabolites and bacterial products that act as signaling molecules, binding to receptors in the intestine and in other metabolically active organs. These signals lead to changes in appetite, gut motility, energy uptake and storage, and also energy expenditure which results in a net positive energy balance and weight gain. Obese humans and rodents have an altered gut microbiota composition with less diversity than in lean controls, and microbiota transplantation transfers this obese phenotype, suggesting that the gut microbiota plays a role in the development of obesity. Further studies are needed to determine how the function of the gut microbiota can be altered to obtain long-term beneficial metabolic effects.

\section{Acknowledgments}

We thank Anna Hallén for assistance with the figure. The authors are supported by grants from the Swedish Research Council, the Åke Wiberg Foundation, the Magnus Bergvall Foundation, the Wilhelm and Martina Lundgren foundation, and an international starting grant from the Sahlgrenska Academy.

\section{References}

1 Sender R, Fuchs S, Milo R: Are we really vastly outnumbered? Revisiting the ratio of bacterial to host cells in humans. Cell 2016;164: 337-340.

2 Gill SR, Pop M, Deboy RT, Eckburg PB, Turnbaugh PJ, Samuel BS, Gordon JI, Relman DA, Fraser-Liggett CM, Nelson KE: Metagenomic analysis of the human distal gut microbiome. Science 2006;312:1355-1359.

3 Backhed F, Roswall J, Peng Y, Feng Q, Jia H, Kovatcheva-Datchary P, et al: Dynamics and stabilization of the human gut microbiome during the first year of life. Cell Host Microbe 2015; 17:690-703.

4 David LA, Maurice CF, Carmody RN, Gootenberg DB, Button JE, Wolfe BE, Ling AV, Devlin AS, Varma Y, Fischbach MA, Biddinger SB, Dutton RJ, Turnbaugh PJ: Diet rapidly and reproducibly alters the human gut microbiome. Nature 2014;505:559-563.

5 Goodrich JK, Waters JL, Poole AC, Sutter JL, Koren O, Blekhman R, Beaumont M, Van Treuren W, Knight R, Bell JT, Spector TD, Clark AG, Ley RE: Human genetics shape the gut microbiome. Cell 2014;159:789-799.

6 Donaldson GP, Lee SM, Mazmanian SK: Gut biogeography of the bacterial microbiota. Nat Rev Microbiol 2016;14:20-32.

7 Li J, Jia H, Cai X, Zhong H, Feng Q, Sunagawa $S$, et al: An integrated catalog of reference genes in the human gut microbiome. Nat Biotechnol 2014;32:834-841.

8 Ley RE, Turnbaugh PJ, Klein S, Gordon JI: Microbial ecology: human gut microbes as- sociated with obesity. Nature 2006;444:10221023.

9 Turnbaugh PJ, Ley RE, Mahowald MA, Magrini V, Mardis ER, Gordon JI: An obesityassociated gut microbiome with increased capacity for energy harvest. Nature 2006;444: 1027-1031.

10 Turnbaugh PJ, Hamady M, Yatsunenko T, Cantarel BL, Duncan A, Ley RE, Sogin ML, Jones WJ, Roe BA, Affourtit JP, Egholm M, Henrissat B, Heath AC, Knight R, Gordon JI: A core gut microbiome in obese and lean twins. Nature 2009;457:480-484.

11 Duncan SH, Lobley GE, Holtrop G, Ince J, Johnstone AM, Louis P, Flint HJ: Human colonic microbiota associated with diet, obesity and weight loss. Int J Obes (Lond) 2008;32: 1720-1724.

12 Le Chatelier E, Nielsen T, Qin J, Prifti E, Hildebrand F, Falony G, et al: Richness of human gut microbiome correlates with metabolic markers. Nature 2013;500:541-546.

13 Ridaura VK, Faith JJ, Rey FE, Cheng J, Duncan AE, Kau AL, et al: Gut microbiota from twins discordant for obesity modulate metabolism in mice. Science 2013;341:1241214.

14 Backhed F, Ding H, Wang T, Hooper LV, Koh GY, Nagy A, Semenkovich CF, Gordon JI: The gut microbiota as an environmental factor that regulates fat storage. Proc Natl Acad Sci USA 2004;101:15718-15723.

15 McNeil NI: The contribution of the large intestine to energy supplies in man. Am J Clin Nutr 1984;39:338-342.
16 Backhed F, Manchester JK, Semenkovich CF, Gordon JI: Mechanisms underlying the resistance to diet-induced obesity in germ-free mice. Proc Natl Acad Sci USA 2007;104:979984

17 Kubeck R, Bonet-Ripoll C, Hoffmann C, Walker A, Muller VM, Schuppel VL, Lagkouvardos I, Scholz B, Engel KH, Daniel H, Schmitt-Kopplin P, Haller D, Clavel T, Klingenspor M: Dietary fat and gut microbiota interactions determine diet-induced obesity in mice. Mol Metab 2016;5:1162-1174.

18 Suarez-Zamorano N, Fabbiano S, Chevalier C, Stojanovic O, Colin DJ, Stevanovic A, et al: Microbiota depletion promotes browning of white adipose tissue and reduces obesity. Nat Med 2015;21:1497-1501.

19 Collins SM, Surette M, Bercik P: The interplay between the intestinal microbiota and the brain. Nat Rev Microbiol 2012;10:735-742.

20 Koda S, Date Y, Murakami N, Shimbara T, Hanada T, Toshinai K, Niijima A, Furuya M, Inomata $\mathrm{N}$, Osuye $\mathrm{K}$, Nakazato $\mathrm{M}$ : The role of the vagal nerve in peripheral PYY3-36-induced feeding reduction in rats. Endocrinology 2005; 146:2369-2375.

21 Schele E, Grahnemo L, Anesten F, Hallen A Backhed F, Jansson JO: The gut microbiota reduces leptin sensitivity and the expression of the obesity-suppressing neuropeptides proglucagon (GCG) and brain-derived neurotrophic factor (BDNF) in the central nervous system. Endocrinology 2013;154:36433651. 
22 Wichmann A, Allahyar A, Greiner TU, Plovier $\mathrm{H}$, Lunden GO, Larsson T, Drucker DJ, Delzenne NM, Cani PD, Backhed F: Microbial modulation of energy availability in the colon regulates intestinal transit. Cell Host Microbe 2013;14:582-590.

23 Bucinskaite V, Tolessa T, Pedersen J, Rydqvist B, Zerihun L, Holst JJ, Hellstrom PM: Receptor-mediated activation of gastric vagal afferents by glucagon-like peptide- 1 in the rat. Neurogastroenterol Motil 2009;21:978.

24 Flak JN, Myers MG Jr: Minireview: CNS mechanisms of leptin action. Mol Endocrinol 2016;30:3-12.

25 Thaler JP, Yi CX, Schur EA, Guyenet SJ, Hwang BH, Dietrich MO, et al: Obesity is associated with hypothalamic injury in rodents and humans. J Clin Invest 2012;122:153-162.

26 Valdearcos M, Robblee MM, Benjamin DI, Nomura DK, Xu AW, Koliwad SK: Microglia dictate the impact of saturated fat consumption on hypothalamic inflammation and neuronal function. Cell Rep 2014;9: 2124-2138.

27 Erny D, Hrabe de Angelis AL, Jaitin D, Wieghofer P, Staszewski O, David E, et al: Host microbiota constantly control maturation and function of microglia in the CNS. Nat Neurosci 2015; 18:965-977.

28 Braniste V, Al-Asmakh M, Kowal C, Anuar F, Abbaspour A, Toth M, Korecka A, Bakocevic N, Ng LG, Kundu P, Gulyas B, Halldin C, Hultenby K, Nilsson H, Hebert H, Volpe BT, Diamond B, Pettersson S: The gut microbiota influences blood-brain barrier permeability in mice. Sci Transl Med 2014;6:263ra158.

29 Tremaroli V, Backhed F: Functional interactions between the gut microbiota and host metabolism. Nature 2012;489:242-249.

30 Caesar R, Nygren H, Oresic M, Backhed F: Interaction between dietary lipids and gut microbiota regulates hepatic cholesterol metabolism. J Lipid Res 2016;57:474-481.

31 Brown AJ, Goldsworthy SM, Barnes AA, Eilert MM, Tcheang L, Daniels D, et al: The orphan $G$ protein-coupled receptors GPR41 and GPR43 are activated by propionate and other short chain carboxylic acids. J Biol Chem 2003;278:11312-11319.
32 Kimura I, Ozawa K, Inoue D, Imamura T, Kimura K, Maeda T, et al: The gut microbiota suppresses insulin-mediated fat accumulation via the short-chain fatty acid receptor GPR43. Nat Commun 2013;4:1829.

33 Samuel BS, Shaito A, Motoike T, Rey FE, Backhed F, Manchester JK, Hammer RE, Williams SC, Crowley J, Yanagisawa M, Gordon JI: Effects of the gut microbiota on host adiposity are modulated by the short-chain fattyacid binding G protein-coupled receptor, GPR41. Proc Natl Acad Sci USA 2008;105: 16767-16772.

34 Xiong Y, Miyamoto N, Shibata K, Valasek MA, Motoike T, Kedzierski RM, Yanagisawa M: Short-chain fatty acids stimulate leptin production in adipocytes through the $\mathrm{G}$ protein-coupled receptor GPR41. Proc Natl Acad Sci USA 2004;101:1045-1050.

35 Psichas A, Sleeth ML, Murphy KG, Brooks L, Bewick GA, Hanyaloglu AC, Ghatei MA, Bloom SR, Frost G: The short chain fatty acid propionate stimulates GLP-1 and PYY secretion via free fatty acid receptor 2 in rodents. Int J Obes (Lond) 2015;39:424-429.

36 Donohoe DR, Garge N, Zhang X, Sun W, O'Connell TM, Bunger MK, Bultman SJ: The microbiome and butyrate regulate energy metabolism and autophagy in the mammalian colon. Cell Metab 2011;13:517-526.

37 Osaka T, Endo M, Yamakawa M, Inoue S: Energy expenditure by intravenous administration of glucagon-like peptide- 1 mediated by the lower brainstem and sympathoadrenal system. Peptides 2005;26:1623-1631.

38 Secher A, Jelsing J, Baquero AF, HecksherSorensen J, Cowley MA, Dalboge LS, et al: The arcuate nucleus mediates GLP-1 receptor agonist liraglutide-dependent weight loss. J Clin Invest 2014;124:4473-4488.

39 Wahlstrom A, Kovatcheva-Datchary P, Stahlman M, Khan MT, Backhed F, Marschall HU: Induction of farnesoid $\mathrm{X}$ receptor signaling in germ-free mice colonized with a human microbiota. J Lipid Res 2017;58:412-419.

40 Thomas C, Gioiello A, Noriega L, Strehle A, Oury J, Rizzo G, et al: TGR5-mediated bile acid sensing controls glucose homeostasis. Cell Metab 2009;10:167-177.
41 Sayin SI, Wahlstrom A, Felin J, Jantti S, Marschall HU, Bamberg K, Angelin B, Hyotylainen T, Oresic M, Backhed F: Gut microbiota regulates bile acid metabolism by reducing the levels of tauro-beta-muricholic acid, a naturally occurring FXR antagonist. Cell Metab 2013;17:225-235.

42 Parseus A, Sommer N, Sommer F, Caesar R, Molinaro A, Stahlman M, Greiner TU, Perkins R, Backhed F: Microbiota-induced obesity requires farnesoid X receptor. Gut 2017; 66:429-437.

43 Mawe GM, Hoffman JM: Serotonin signalling in the gut-functions, dysfunctions and therapeutic targets. Nat Rev Gastroenterol Hepatol 2013;10:473-486.

44 Sumara G, Sumara O, Kim JK, Karsenty G: Gut-derived serotonin is a multifunctional determinant to fasting adaptation. Cell Metab 2012;16:588-600.

45 Reigstad CS, Salmonson CE, Rainey JF 3rd, Szurszewski JH, Linden DR, Sonnenburg JL, Farrugia G, Kashyap PC: Gut microbes promote colonic serotonin production through an effect of short-chain fatty acids on enterochromaffin cells. FASEB J 2015;29:13951403.

46 Cani PD, Amar J, Iglesias MA, Poggi M, Knauf C, Bastelica D, et al: Metabolic endotoxemia initiates obesity and insulin resistance. Diabetes 2007;56:1761-1772.

47 Cani PD, Bibiloni R, Knauf C, Waget A, Neyrinck AM, Delzenne NM, Burcelin R: Changes in gut microbiota control metabolic endotoxemia-induced inflammation in high-fat diet-induced obesity and diabetes in mice. Diabetes 2008;57:1470-1481.

48 Caesar R, Tremaroli V, Kovatcheva-Datchary P, Cani PD, Backhed F: Crosstalk between gut microbiota and dietary lipids aggravates WAT inflammation through TLR signaling. Cell Metab 2015;22:658-668.

49 Ghoshal S, Witta J, Zhong J, de Villiers W, Eckhardt E: Chylomicrons promote intestinal absorption of lipopolysaccharides. J Lipid Res 2009;50:90-97.

50 Breton J, Tennoune N, Lucas N, Francois M, Legrand R, Jacquemot J, et al: Gut commensal E. coli proteins activate host satiety pathways following nutrient-induced bacterial growth. Cell Metab 2016;23:324-334. 